\title{
Tale of the Swan-Ganz (Pulmonary Artery) Catheter
}

\author{
Fayaz A Hakim, $M D^{1}$ and Faroque Ahmad Khan, $\mathrm{MB}, \mathrm{MACP}{ }^{2}$ \\ 1Department of Internal Medicine \\ ${ }_{2}^{2}$ Department of Research and Publication, \\ King Fahd Medical City \\ Riyadh, Saudi Arabia
}

\begin{abstract}
Since its introduction in 1970, the Swan-Ganz catheter (SGC) has been used extensively, particularly in critical care units. A recent well-designed randomized clinical trial confirmed earlier suspicions that the widespread use of the SGC caused more harm than good. This account of historical SGC use has some valuable lessons for all practitioners, who at times tend to embrace and overuse the ever-increasing diagnostic or therapeutic modalities without fully assessing the risk/benefit ratio.
\end{abstract}

Key words: Swan-Ganz catheter, pulmonary artery catheter, evidence-based medicine.

I mpressed by a sketch in a physiology textbook that showed a physiologist holding a thin tube that had been put into the heart of a horse through its jugular vein, Werner Frossman, a 25year-old intern, made history in 1929 when he guided a catheter into his own heart through one of his antecubital veins, thus disproving the decades-long myth "Do not touch the heart". Although criticized for his idea of touching the human heart, he pursued his idea by self-experimentation and earned a Nobel

Correspondence should be directed to

Fayaz A. Hakim, MD

Division of Internal Medicine

4th Floor, Main Hospital

King Fahd Medical City

P.O/Box 59046

Riyadh, 11525

Saudi Arabia

Email:fayazhakim@hotmail.com

Telephone: 966-1-2889999 extension 1396

Fax: 966-1-2889999 extension 1395
Prize in $1956 . .^{1}$ We owe present-day knowledge of cardiac physiology, hemodynamics, heat-lung interaction, and catheter-based procedures in the diagnosis and treatment of various cardiac ailments to the great idea and courage of this man.

Physicians caring for critically ill patients in intensive care units (ICUs) were puzzled about how to use this technique to obtain pulmonary capillary wedge pressure, an indirect measurement of left atrial pressure. The issue was how to guide the catheter into the pulmonary artery and then distally into pulmonary capillaries. Swan's critical observation of boats drifting quickly even in presence of a very mild breeze lead to the birth of the balloontipped, flow-directed pulmonary artery catheter (PAC) by Swan and Ganz in 1970.2 The device was accepted widely as a bedside tool for hemodynamic measurements and optimizing tissue oxygenation in critically ill patients in ICU settings with the aim that such intervention would improve outcome in these patients. Presumed that its benefits would be great, and the risk would be the same as that of simple cardiac catheterization, the Swan-Ganz PAC was widely 
accepted and became a part of the management of all critically ill patients admitted to ICUs. While all drug and therapeutic interventions go through intense scrutiny, including clinical trials, before receiving approval for clinical use, such standards are nonexistent for diagnostic and monitoring modalities, as was the case with the Swan-Ganz catheter.

Clinical trials were performed to assess the risk/benefit ratio of the PAC prior to its use and after its introduction in 1970. The device also had a significant impact on the economy. An estimate in the late 1990s revealed that more than a million PACs were used each year in the United States, with an estimated annual cost of $\$ 2$ billion.

Robbins in $1985^{3}$ first raised the issue of overuse and abuse of the PAC. Ever since, small observational studies have reported increased morbidity and mortality associated with the use of the PAC in critically ill patients from time to time. Gore et al ${ }^{4}$ reported a $50 \%$ increase in mortality, Zion et al ${ }^{5}$ reported a mortality rate four times higher, and Blumberg et al ${ }^{6}$ reported a two-fold increase in the mortality rate among catheterized patients as compared to noncatheterized patients. These observations were further supported by a large prospective cohort study by Connors et al in 1996 of almost 6000 patients treated at five medical centers, putting the tool in further dispute. ${ }^{7}$ Subsequent to this growing concern regarding the use of the PAC, more than a dozen randomized clinical trials addressing the safety or efficacy of the PAC were conducted to eliminate the deficiencies of previously reported observational and cohort studies and to resolve the controversies surrounding the use of the PAC. Most of these small randomized clinical trials yielded results discouraging the use of the PAC in common ICU settings and put the use of this empiric and invasive tool further in doubt. Sandham et al, ${ }^{8}$ Richard et al, ${ }^{9}$ and Binanay et al10 in three different randomized clinical trials conducted on elderly high-risk surgical patients, patients with shock and acute respiratory distress syndrome, and patients with congestive heart failure, respectively, reported no significant differences in the mortality rate in patients managed with a PAC than those without, yet there was no benefit from its use in those who had it. Shah et al ${ }^{11}$ in 2005 conducted a meta-analysis of 13 randomized clinical trials and came up with the conclusion that use of the PAC neither increases overall mortality nor confers ben- efit. Since these randomized clinical trials were inadequate in terms of methodology, sample selection and size and lack of comparison groups etc., the question remained unanswered. Wheeler et al. in 2006 finally came up with a well-designed randomized control trial of the management of acute lung injury to evaluate the benefits and risks of PACs, addressing all uncertainties. ${ }^{2}$ This trial was sponsored by the National Institutes of Health (NIH) and included 1000 patients with established acute lung injuries to compare hemodynamic management guided by a PAC with hemodynamic management guided by a central venous catheter (CVC) using explicit management protocols. The conclusion drawn from this study was that PAC-guided therapy did not improve overall survival or organ function but was associated with more complications than CVC-guided therapy. Now looking at the strengths of the study, it appears at this point that the use of PACs in guiding fluid therapy in critically ill patients with sepsis, acute respiratory distress syndrome (ARDS), and congestive heart failure is not indicated both on ethical and medical grounds. However, its role in conditions that were not included in the studies and where PAC is indicated cannot be underestimated, especially in the diagnosis, treatment, and monitoring efficacy of treatment in patients with pulmonary artery hypertension.

Pulmonary artery catheters in ICU settings are commonly used to guide fluid therapy into critically ill patients with ARDS, pulmonary edema, congestive heart failure, sepsis, and during high risk surgeries. In modern times, evidence-based medicine is in demand in all fields, including the ICU. Now, level A evidence exists that shows PACs do not improve overall survival rates in critically ill patients admitted to ICUs with the above-mentioned conditions. After Wheeler's study in 2006, its use in common ICU settings, as mentioned above, has been stopped in the West and Europe.

No data is available regarding the extent of use of PACs in critically ill patients in ICU settings in most parts of the world. Availability of the Swan-Ganz catheter is never a problem in most of the hospitals in Saudi Arabia, and a majority of the physicians in Saudi Arabia are trained in the United States and Canada, where the use of the PAC was routine with most critically ill patients admitted to an ICU. Because of these two factors, it appears there may be 
a tendency to overuse the PAC. Similar factors could also account for continued inappropriate use of this device in other parts of the Middle East. Since there is clear evidence from a well-designed randomized clinical trial that there is no benefit from routine use of a PAC in critically ill patients and measurements obtained from the central venous catheter are equally reliable, it appears prudent that ICU physicians should be warned against routine use of the PAC, especially when fluid therapy is an issue. This will not only prevent critically ill patients from undergoing unnecessary procedures and procedure-related complications but also will have a significant favorable impact on hospital resources and economy.

In closing, the Swan-Ganz pulmonary artery catheter serves as an important lesson in the appropriate use of modern invasive and noninvasive equipment and reinforces the need for appropriate studies to evaluate the risk and benefit ratio before embarking on widespread use of the ever-increasing new medical monitoring, testing, and treating devices.

\section{References}

1. Lawrence KA. Who goes first? The story of self experimentation in medicine. Berkeley, University of California Press.1998;39-52.

2. Swan HJ, Ganz W, Forrester J, et al. Catheterization of the heart in man with use of flow directed balloontipped catheter. N Engl J Med 1970;283:447-51.

3. Robin ED. The cult of the Swan-Ganz catheter. Overuse and abuse of pulmonary flow catheters. Ann Intern Med 1985;103:445-9.

4. Gore JM, Goldberg RJ, Spodick DH, et al. A commu- nity-wide assessment of the use of pulmonary artey catheters in patients with acute myocardial infarction. Chest. 1987;92:721-7.

5. Zion MM, Balkin J, Rosenmann, et al. Use of pulmonary artey catheters in patients with acute myocardial infarction: analysis of experience in 5841 patients in SPRINT registry. Chest. 1990;98:1331-5.

6. Blumberg MS, Binns GS. Swan-Ganz catheter use and mortality in myocardial infarction patients. Health Care Financ Rev. 1994;15:91-103.

7. Connors AF Jr., Speroff T, Dawson NV, et al. The effective of right heart catheterization in the initial care of critically ill patients. JAMA 1996;276:889-97.

8. Sandham JD, Hull RD, Brant RF, et al. A randomized, controlled trial of the use of pulmonary-artery catheters in high-risk surgical patients. N Engl J Med. 2003;348:5-14.

9. Richard C, Warszawski J, Anguel N, et al. Early use of pulmonary artery catheter and outcomes in patients with shock and acute respiratory distress syndrome: a randomized controlled trial. JAMA. 2003;290:2713-20.

10. Binanay C, Califf RM, Hasselblad V, et al. Evaluation study of congestive heart failure and pulmonary artery catheterization effectiveness: the ESCAPE trial. JAMA. 2005;294:1625-33.

11. Shah MR, Hasselblad V, Stevenson LW, et al. Impact of pulmonary artery catheter in critically ill patients: a metaanalysis of randomized clinical trials. JAMA. 2005;294:1664-70.

12. Wheeler P, Bernard GR, Thompson BT. Pulmonary-artery versus central venous catheter to guide treatment for acute lung injury. N Engl J Med. 2006;354(21):2213-24. 